Document downloaded from:

http://hdl.handle.net/10251/63950

This paper must be cited as:

Sanchez Belenguer, C.; Vendrell Vidal, E.; Sánchez López, M.; Díaz Marín, MDC.; AuraCastro, E. (2015). Automatic production of tailored packaging for fragile archaeological artifacts. Journal on Computing and Cultural Heritage. 8(3):17:1-17:11. doi:10.1145/2716324.

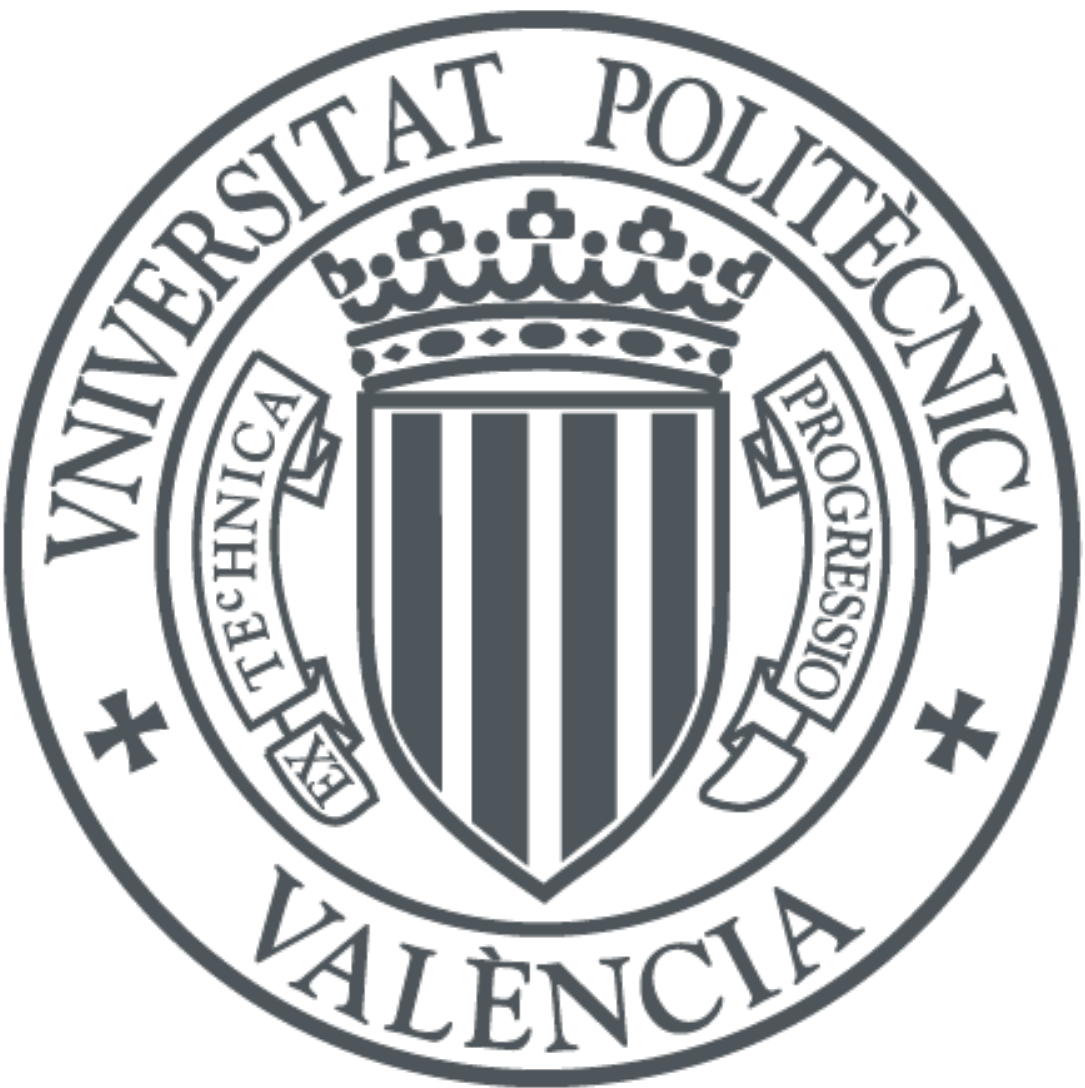

The final publication is available at http://dx.doi.org/10.1145/2716324

Copyright ACM

Additional Information 


\title{
Automatic Production of Tailored Packaging for Fragile Archaeological Artifacts
}

\author{
CARLOS SÁNCHEZ-BELENGUER, ai2 - UPV, ITU - Joint Research Centre \\ EDUARDO VENDRELL-VIDAL, ai2 - Universitat Politècnica de València \\ MIGUEL SÁNCHEZ-LÓPEZ, DISCA - Universitat Politècnica de València \\ CARMEN DÍAZ-MARÍN and ELVIRA AURA-CASTRO, IRP - Universitat Politècnica de València
}

\begin{abstract}
This paper deals with storage and transportation of fragile heritage objets. The main goal is to create an ad-hoc packaging software solution by combining the use of 3D scanning and 3D milling technologies. Traditionally, packaging for this kind of objects is made manually, creating custom supports and boxes adapted to the specific needs and particularities of each case. This process is time-consuming and fully dependent on the conservator's skills. On the other hand, the proposed solution provides a faster execution and a standard solution adapted to every object. The process starts with the acquisition of 3D digital models of the original artifacts and uses an interactive GPU-accelerated algorithm to assist the conservator in the construction of the package. The final result is a CNC program that is sent to a low cost milling machine to produce the housing for the selected artifact. Unlike 3D printers, milling machines allow working with lots of different materials, which is a key aspect for this kind of packages: chemical stability and shock absorption are mandatory. Given that, during the design process, original artifacts are only manipulated in the acquisition stage, risks of damaging them are also reduced. Since the whole process is considered in the same application, intermediate calculations are performed considering manufacturing and user-defined restrictions. This way, together with a GPU implementation, achieved results are very fast providing a real-time application with visual feed-back.

Categories and Subject Descriptors: J.6 [Computer Applications]: Computer-Aided Engineering-Computer-aided manufacturing (CAM); I.3.5 [Computer Graphics]: Computational Geometry and Object Modeling-Curve, surface, solid, and object representations; Modeling packages
\end{abstract}

General Terms: Algorithms

Additional Key Words and Phrases: Packaging, Conservation, Heritage, GPU, CNC

ACM Reference Format:

ACM J. Comput. Cult. Herit. 1, 1, Article 1 (January 1), 11 pages.

DOI : http://dx.doi.org/10.1145/0000000.0000000

Author's address: C. Sánchez, Institute for Transuranium Elements. European Commission, Joint Research Centre (JRC). Via Enrico Fermi 2749. Ispra (VA), Italy; email: carsanb1@doctor.upv.es; E. Vendrell, Avenida de los Naranjos, s/n, 46022 Valencia (Spain); email: even@upv.es; M. Sánchez, Avenida de los Naranjos, s/n, 46022 Valencia (Spain); email: misan@upvnet.upv.es; C. Díaz, Avenida de los Naranjos, s/n, 46022 Valencia (Spain); email: madiama@posgrado.upv.es; E. Aura, Avenida de los Naranjos, s/n, 46022 Valencia (Spain); email: eaura@crbc.upv.es

Permission to make digital or hard copies of part or all of this work for personal or classroom use is granted without fee provided that copies are not made or distributed for profit or commercial advantage and that copies show this notice on the first page or initial screen of a display along with the full citation. Copyrights for components of this work owned by others than ACM must be honored. Abstracting with credit is permitted. To copy otherwise, to republish, to post on servers, to redistribute to lists, or to use any component of this work in other works requires prior specific permission and/or a fee. Permissions may be requested from Publications Dept., ACM, Inc., 2 Penn Plaza, Suite 701, New York, NY 10121-0701 USA, fax +1 (212) 869-0481, or permissions@acm.org.

(c) 1 ACM $1556-4673 / 1 / 01-A R T 1 ~ \$ 15.00$

DOI : http://dx.doi.org/10.1145/0000000.0000000

ACM Journal on Computing and Cultural Heritage, Vol. 1, No. 1, Article 1, Publication date: January 1. 
ORIGINAL ARTIFACT

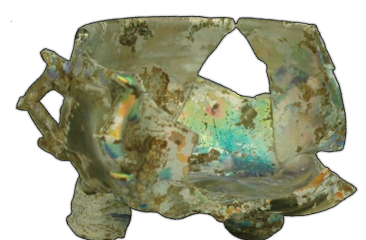

3D MODEL

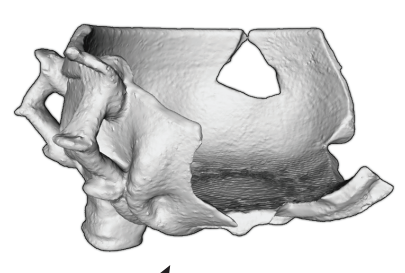

HOUSING

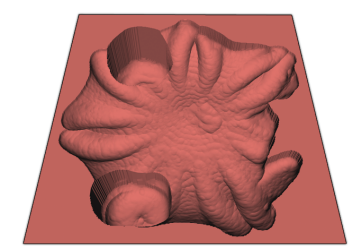

\section{CNC TRAJECTORIES}

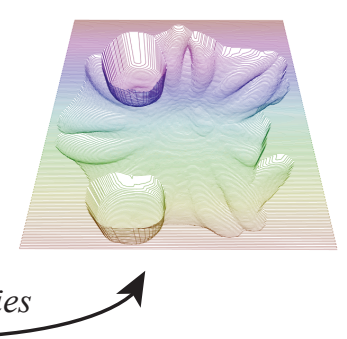

Fig. 1. Proposed stages for the automatic production of tailored packaging, from the original artifact to the CNC trajectories used by the milling machine. Each stage takes as input data the results from the previous one, together with a set of parameters introduced by the conservator that allow generating the final product according to the specifics of each artifact/milling machine.

\section{INTRODUCTION}

The building of the housing structure of an artifact has been traditionally a manual process requiring the presence and continuous manipulation of the original object. These techniques require a skilled hand and a good amount of time for trial and error until the shape of the housing is just perfect. Along the process, the need of handling the original artifact for every check performed during the carving process is a source of damage risk.

Institutions responsible for heritage are progressively adopting new digital techniques to their arsenal of tools that bring new ways of performing certain tasks. Computer-aided manufacturing (CAM) provides a way of automating the carving process of housing structures.

This paper presents a set of algorithms developed to cover the entire manufacturing process of this kind of structures, from the packaging design to the milling stage of its parts. Using a 3D digital model of the the original artifact as input data, the proposed technique selects the best orientation and creates the files required for machining the different parts of the packaging.

Not only this process will produce a suitable packaging every time, but the artifact will no longer be used while the housing is produced, which removes part of the risk of the operation. Required input data are only 3D scans of the artifacts, which may already be available in some institutions (i.e: Smithsonian Institution).

In each case, the proposed packaging will be really suitable if it is supervised by the conservator. This professional might decide when is possible to accept the automatic solution depending of many factors like the artifact's state of preservation, its fragility or thickness, among others factors. It also corresponds to him to decide other issues that affect the packaging, like the foam type to use or other factors like if the artifact has to be completely enclosed by the packaging or not. Conditions of storage, transit or transport situations must be taken as well into account.

\subsection{Overview}

The housing manufacturing process proposed in this paper is divided in four different stages detailed below, and illustrated in Figure 1:

-Acquisition: using a triangulation 3D laser scanner and a turntable, artifacts are acquired and stored as 3D B-Rep meshes. These models will be provided as input data to the algorithm, but can also be used for other applications (e.g. creating a database of artifacts, developing visualization tools...).

-Modeling: according to the input 3D model, a pose that maximizes contact surface between the artifact and the housing is calculated automatically and a software tool generates a 3D model of 
the housing that considers the physical restrictions introduced by the milling hardware. Results are computed very fast (even in real-time), and accuracy is extremely high.

- Trajectory calculation: from the 3D model of the housing generated in the previous stage, and considering some manufacturing parameters (radius and length of the milling tool), a set of trajectories for the milling machine are calculated, and a G-Code program is saved into a file.

-Milling: once trajectories are calculated the G-Code program that was generated in the previous stage is sent to a low cost milling machine that carves a foam block and produces the final housing.

Major contributions presented in this paper are: (1) the development of an automatic procedure that covers the entire manufacturing process of tailor-made packaging: from modeling to milling and (2) an automatic technique to orient the artifacts in order to maximize the contact area between the package and the surface of the artifact.

\subsection{Document Organization}

This paper is organized as follows: Section 2 reviews existing related work, Section 3 presents the proposed technique in detail (automatic model generation, pose estimation and milling trajectories computation), Section 4 presents some results and, finally, Section 5 draws some conclusions and suggests future improvements to the proposed technique.

\section{RELATED WORK}

Artifact packaging has evolved from simple boxes with padding material to more complex compact cushioning. These cushioning are placed by adapting them to inner holes in a box container, and their main function is protection against shock and vibration.

From this packaging technique (an artifact inside a single or double box, protected by a set of cushioning layers), different approaches appear trying to design specific housings that fit the particularities of each kind of artifact. Several packaging designs, together with their cushioning systems, are described in works like [Stolow 1987] and [Johnson 1999].

A very common material used for these kind of tailored housings is the rigid foam, since it can be cut in order to fit the shape of the artifact to protect. Foam is also used for storage, given that it allows holding artifacts in a very stable and safe way, which is very important when working with extremely fragile materials.

The most common types of housings with rigid foam are:

-Rigid foam template packing: which consists on using struts or inner supports that enclose the artifact inside a set of foam layers fixed in the box container by lateral slots [Stolow 1981].

-Cavity packing: the silhouette of the artifact is cut into the foam, creating a hole where the artifact is introduced and fixed. It is important not to tighten it excessively, so it can be easily extracted [Bauer 1993].

-Cradle mounts: used for concave and non stable artifacts, consist on cutting out multiple blocks of rigid foam to create a base that provides better stability [Murdock and Johnson 2001].

Figure 2 shows these three kinds of housing structures.

\subsection{Housing manufacturing}

In the last years there have been significant advances in the housing design process and cushioning systems specifically created for heritage conservation. This way, in [Marcon 1991] reference tables were designed to help with the calculation of optimal housings and, in [Marcon and Strang 1999] the Canadian Conservation Institute created a software tool for the automatic external cushioning design 

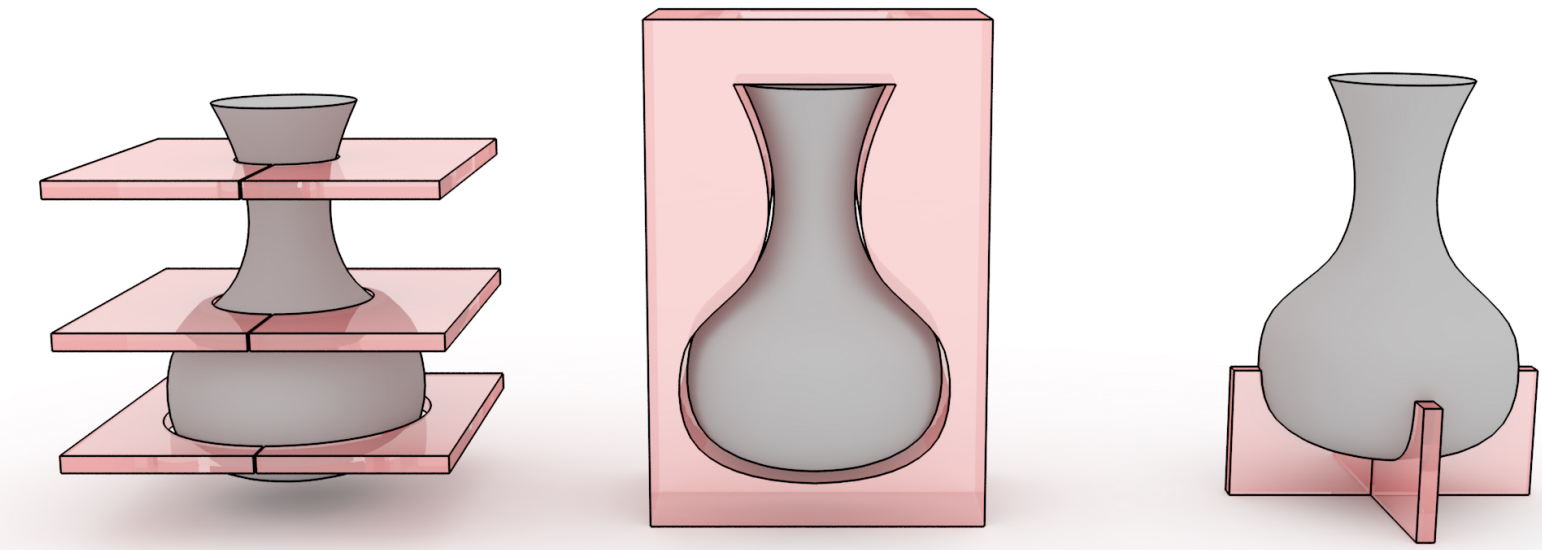

Fig. 2. Different types of tailored housings. (left) Rigid foam template packing. (center) Cavity packing. (right) Cradle mounts.

for fragile artifacts. More recently, in [Marcon 2014b] a guide for building housings for fragile objects and safe shipments is introduced and, in [Marcon 2014a] a deep study in the physical forces involved in deterioration and their consequences is presented.

Thanks to these works, fragile archaeological artifacts (like glass) can nowadays be packed for storage and/or transportation without risks [Fulton and Rossi-Wilcox 2008]. Studies on cushioning systems have identified the most adequate materials [Tetreault and Williams 1993] for these purposes. One of the materials that stands out above the rest recently is polythene foam, given its vibration absorption capabilities and its chemical stability [Schlichting and Institute 1994]. However, working with polythene foam is not easy or fast: it has to be manually cut following the contour and volume of the artifact to protect [Aboe 2012] and, when its shape is complex, results tend to be very inaccurate.

Nowadays, with the rise of the 3D printing technology, there are new possibilities in the field of tailored packaging. In [Mohamed and Mahmoud 2012] their influence is analyzed and the beginning of a revolution in this field is highlighted. In [Medeiros-S et al. 2012] heritage applications are presented and a case of study about artifact packaging is used to evidence the potential advantages of this technology. In this work, an additive 3D printer is used to create light linen structures.

Despite the advantages of 3D printers, the limitations imposed by the materials that can be used make CNC (Computer Numerical Control) cutting machines more suitable for packaging purposes. This way, in [Gallup and Harlow 2006] a study for the Yale University Art Gallery is presented, where custom housings are created using CNC machines to cut rigid foam blocks. These housings are created from 3D models obtained with a triangulation laser scanner. More recently, and in a similar way, the Smithsonian Institution of Washington is using a milling machine on polythene foam to make the housings for contemporary artwork [Smithsonian 2014]. An overview on 3D acquisition techniques for Cultural Heritage can be found in [Pieraccini et al. 2001].

\section{TECHNIQUE}

The proposed technique automatically models a solid 3D mesh of the package, taking advantage of modern GPU computing features. During the modeling stage, geometry is built considering milling procedures/restrictions so the resulting housing is easy to mechanize. Given that all stages in the process are considered, an efficient and cost-effective solution is provided. 
This approach considerably differs from the one presented in [Medeiros-S et al. 2012] in two main aspects: (1) ours is based on CNC milling technology, that allows using a bigger variety of materials, more suitable to archaeological purposes (chemical stability is a key factor). Also, CNC milling machines are cheaper than plaster based powder 3D printers, allowing us to provide a low-cost solution. (2) The housing topology proposed in [Medeiros-S et al. 2012] is based on low density structures (tetrahedral-octahedral frames) which are hard to compute, provide a limited contact surface, and very hard to print (actually, the original paper shows some printing failures). Our technique proposes an easy-to-calculate and easy-to-print structure that maximizes the contact area.

\subsection{Automatic Generation of the Tailored Packaging}

The goal of this stage consists on automatically calculating a structured 3D model of the housing for the artifact. The input data for the algorithm are the acquired 3D model of the artifact, together with a set of customizable parameters introduced by the conservator. The result is a 3D mesh that wraps the original object, and that considers the restrictions imposed by both, the milling machine and the conservator. Restrictions related to the manufacturing process are mostly a consequence of the degrees of freedom that the milling machine offers and the accuracy achieved. In our case, we are using a typical 3 DOF cartesian milling machine, where the tool moves freely being always perpendicular to the $X-Y$ plane. Parameters introduced by the conservator establish the dimensions of the package, and the desired offset to prevent creating a too narrow housing. Figure 3 shows this parameters.

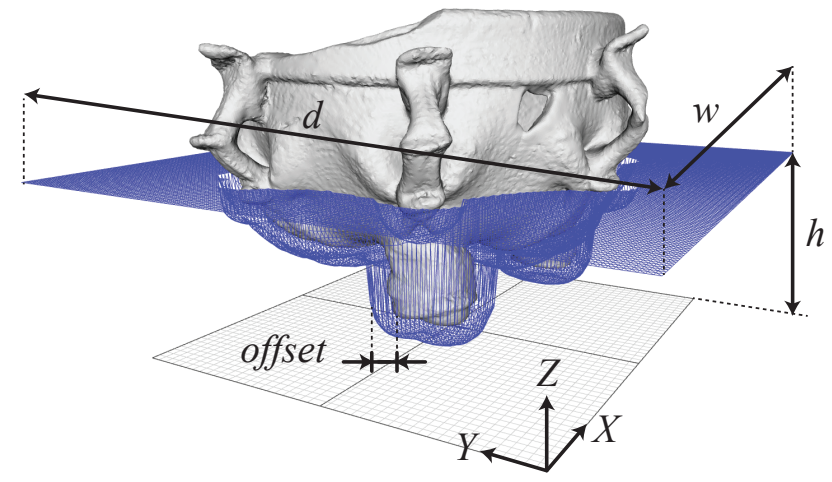

Fig. 3. Parameters considered for automatically generating the housing.

Considering a $3 \mathrm{DOF}$ cartesian milling machine, the working space of the proposed algorithm can be represented as a discrete matrix $P_{u \times v}$ where each sample $p_{i, j}$ stores the coordinates of a 3D point described as: $\left(i * \frac{w}{u}, j * \frac{d}{v}, P(i, j)\right)$, being $w$ and $d$ the width and depth selected by the conservator for the housing of the artifact, respectively, and $u$ and $v$ the resolution of the mesh in both axis.

In order to calculate the $Z$ values for all the points, and considering that the final 3D model will be convex when viewed from the $Z$ axis, the orthogonal projective distances between the base of the housing and the surface of the artifact can calculated by using the GPU (Graphic Processor Unit) computing capabilities [Sanchez-Belenguer and Vendrell-Vidal 2012].

Once the coordinates of each point have been calculated, a global offset is applied to prevent having a too narrow housing for the original artifact. To efficiently do so, we take advantage of the planar representation chosen: since convexity from the $Z$ axis has to be maintained, a convolution kernel can be applied to each point in the matrix, so $Z$ values are updated according to their local neighborhood. 


\subsection{Automatic Orientation of the Artifacts}

In order to fully automatize the manufacturing process, artifacts have to be oriented in a convenient way without user intervention. To do so, proper values for $\theta_{X}$ and $\theta_{Y}$ have to be found. Given the proposed reference frame (Figure 3), $\theta_{Z}$ values do not affect the final result, since axis $Z$ is aligned with the milling direction.

We define the quality of an orientation $\left(\theta_{X}, \theta_{Y}\right)$ according to two criteria: (1) the total contact surface between the housing and the artifact and (2) the degree of perpendicularity between the contact surface and the housing. The bigger these values are, the better the orientation is.

To decide which pose is the most appropriate for packaging purposes, we take advantage of the previously introduced GPU algorithm to perform a grid search optimization. This way, by exhaustively evaluating all pairs of orientations for both axes (iterating with a fixed step value), a cost function is computed for each combination of individual rotations. Then, the pose that maximizes this value is selected as the best one. More formally, the optimization process can be expressed as:

$$
\max _{\theta_{X}, \theta_{Y}} \sum_{t \in T} \frac{1}{2}\left|\left(t_{2}-t_{0}\right) \times\left(t_{2}-t_{1}\right)\right| *\left\langle(0,0,1)^{T}, t_{n}\right\rangle^{2}
$$

where $T$ is the set of triangles returned by the previously introduced algorithm using angles $\theta_{X}$ and $\theta_{Y}, t_{i}$ is the position of the $\mathrm{i}$-th vertex from triangle $t \in T, t_{n}$ corresponds to its normal, the operator $\times$ is the cross product and the operator $\langle a, b\rangle$ corresponds to the dot product between vectors $a$ and $b$.

Notice how, by taking advantage of the GPU-based algorithm, results achieved with the proposed iterative technique are more accurate than using a closed-form solution like PCA analysis. The main reason for this is that occlusions and perpendicularity are taken into account in our technique. Also, thanks to the graphics performance of wired units, execution times are very low, as next section shows.

Additionally, considering that for transportation and storage purposes the artifact should be completely enclosed by the packaging, the optimization process proposed in Equation (1) can be extended by considering $T$ as the union of the set of triangles returned by computing independently the housings for the upper and the lower part of the artifact (milling directions defined as $Z^{+}=(0,0,1)$ and $\left.Z^{-}=(0,0,-1)\right)$. This way, results will ensure that the total contact surface from both sides is maxima. This concept is illustrated in detail in Figure 4.
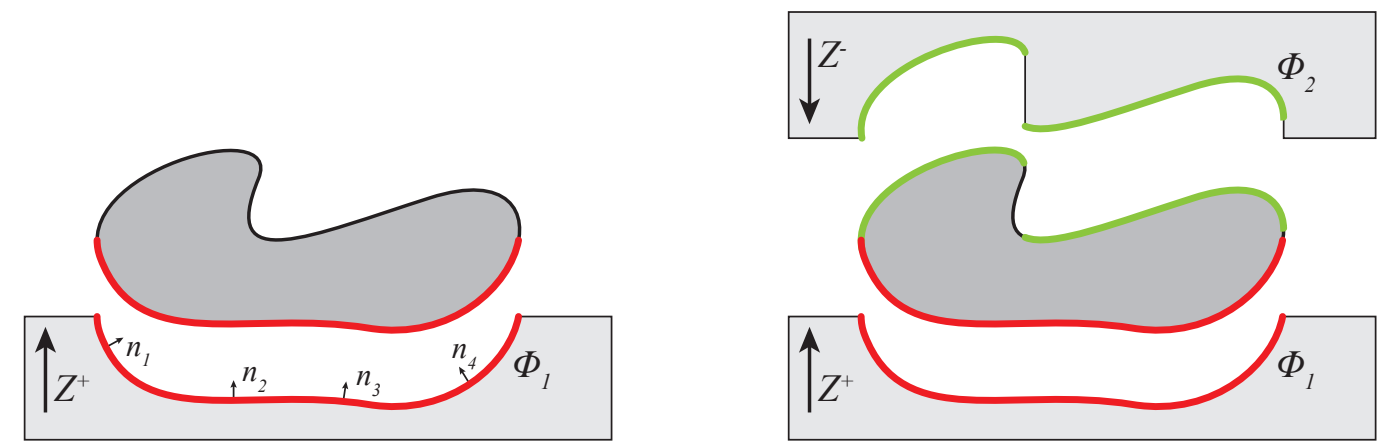

Fig. 4. (left) One-sided packaging of an artifact (represented in darker gray), considering only the $Z^{+}$direction. The red line corresponds to the total contact area (surface $\Phi_{1}$ ), where no occlusions happen. Some local normals are displayed $\left(n_{1 . .4}\right)$, being the area surrounding $n_{2}$ and $n_{3}$ more interesting in terms of perpendicularity with respect to the area surrounding $n_{1}$ and $n_{4}$. (right) Two-sided packaging of the same artifact. Notice how the contact surface $\Phi_{2}$ is not continuous. This situation can only be detected when considering occlusions, and provides more accurate results than closed-form solutions such as PCA analysis. 


\subsection{Milling}

Last stage in the proposed approach deals with the manufacturing of the final tailored package. Materials used for packaging are mostly different types of foams of relatively low density. In order to machine these materials, a light CNC machine can be a low-cost equipment. However, in the long run, most of the cost will come from the cost of the material used for doing the packing.

Taking the input 3D model generated in the previous stage, our milling strategy honors a userdefined overlap percentage and step down values in a zig-zag. Roughing and finishing paths are created, though for some foams roughing phase can be omitted and finishing can be done directly.

Tool-path is calculated as a sequence of points obtained by the intersection the different $X$ axis planes with the packaging 3D mesh. Each one of such planes separated from each other on the $Y$ axis by pass-overlap distance times tool diameter size. If step down value is lower that the stock height, then multiples passes will be needed.

Considering that the previous stage takes into account that the final 3D model has to be milled, point resolution on the $X-Y$ plane is computed according to the pass-overlap distance and the tool diameter. This way, generating the zig-zag trajectories becomes as simple as converting the previous calculated discrete matrix into a set of G-Code commands.

\section{RESULTS}

To illustrate the possibilities of the proposed technique, a complex and fragile archaeological artifact has been selected: a glass made bowl from the 16th-17th century with 3-5 millimeter walls (Figure 1).

During the entire proposed process, the original object was only manipulated in the acquisition stage, to place it over a turning plate. This way, the risk of damaging it was considerably reduced.

Once the artifact was acquired, generating the 3D model for the package was almost immediate: with a real-time GPU-accelerated preview of the parameters, it was only a matter of deciding the proper height for the housing. Computing the final geometry took around 2 seconds, and generating the CNC trajectories took another 2 extra seconds. The manufacturing of the final package took approximately one hour with the low-cost milling machine introduced, no errors were observed during the entire process (which is a common situation with low-cost 3D printers) and no user interaction was required.

The milling tests have been performed using blocks (40 mm height) of extruded polystyrene and polyethylene foam. These materials have been selected because of their low cost and the ease of milling. Also, both materials have been traditionally used for transportation of museum objects.
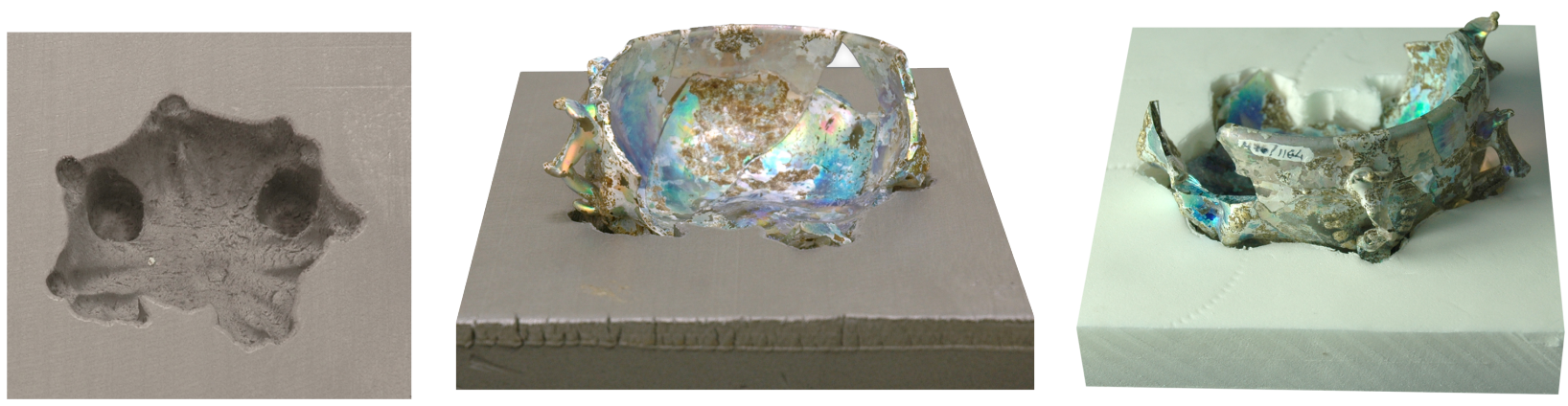

Fig. 5. Achieved result after the complete process. Notice how the final accuracy of the generated package is very high, and the match with the original artifact is perfect, providing a very robust support for storage. In case of transportation purposes, an upper housing could be milled in order to fully enclose the original artifact. (left and center) Results using a polystyrene block. (right) Results using a polyethylene block (PLASTAZOTE@FOAM, type LD45). 
Given that the tool used was long enough ( $60 \mathrm{~mm}$ ), only one pass on the $Z$ axis was necessary. In case the length of the tool were smaller than the height of the block, more extra passes would be needed, and the manufacturing time would be increased.

The final result (Figure 5) is a support/padding with the shape of the artifact, where it is introduced. This foam can be placed inside a box for its transportation and storage.

In order to empirically evaluate the results of the automatic pose estimation algorithm, a set of artifacts with different topologies has been used. All tests have been performed considering a two-sided packaging strategy, with a tool spacing of 0.2 millimeters and a manufacturing error of 1 millimeter. With this set of parameters, extremely precise results can be achieved (perhaps too much detail for low-cost millers). The final size of the package has been automatically calculated in order to provide a constant offset on the sides, and to fully enclose the original object.

Table I summarizes the execution cost for each artifact, together with the number of triangles of its 3D model. As it can be noticed, the complexity of the mesh linearly affects the time required to perform the pose estimation (Bowl2 is a clear example of this fact), whilst the modeling of the package is more affected by the size of the artifact. In this sense, Venus is considerably bigger than the rest of objects, and the calculation time increases up to 19 seconds.

Table I. Time results for multiple fragments.

\begin{tabular}{r|ccc} 
& Triangles & Pose estimation (s.) & Package modeling (s.) \\
\hline \hline Bowl1 & 138.818 & 1,53116 & 6,49399 \\
Bowl2 & 681.702 & 5,50679 & 6,44027 \\
Plate & 400.323 & 3,49614 & 6,31780 \\
Brick & 360.484 & 3,05988 & 1,94527 \\
Cake & 181.564 & 1,79420 & 5,94060 \\
Venus & 371.662 & 3,38959 & 19,10650
\end{tabular}

Figure 6 shows the results of the pose estimation algorithm. In order to facilitate the reading of the images, upper parts of the packaging are not represented. However, results shown in Table I include the time spent in calculating them.

Notice how achieved poses satisfy the proposed maximization criteria of perpendicularity and contact area with the housing. Furthermore, resulting orientations of artifacts (initially loaded in a random pose) correspond to the natural way of storing them: the plate lays over its base (if placed in the opposite way as it is displayed) and the two bowls are standing on their legs. The Venus body lays over its front and back sides, and the two brick pieces (Cake and Brick) are oriented in order to have their two major flat surfaces in contact with the housing.

\section{CONCLUSIONS AND FUTURE WORK}

This paper presents a fully automated solution for the production of tailored packaging for archaeological fragile artifacts. This solution covers all the processes, from the design of packaging linings to the milling of these parts. A software tool has been implemented and evaluated, providing very accurate results, and accelerating the manufacturing process considerably. This application starts from the $3 \mathrm{D}$ scanned model of the artifact and generates a complete housing solution. Then, this solution is manufactured using a 3D axis low-cost milling machine.

A basic algorithm is considered in order to automatically generate the milling code for the CNC machine. This algorithm is based on a sequence of points obtained from the intersection between different $\mathrm{X}$-aligned planes with the 3D model of the artifact, an overlap percentage and step down values in a zig-zag. 

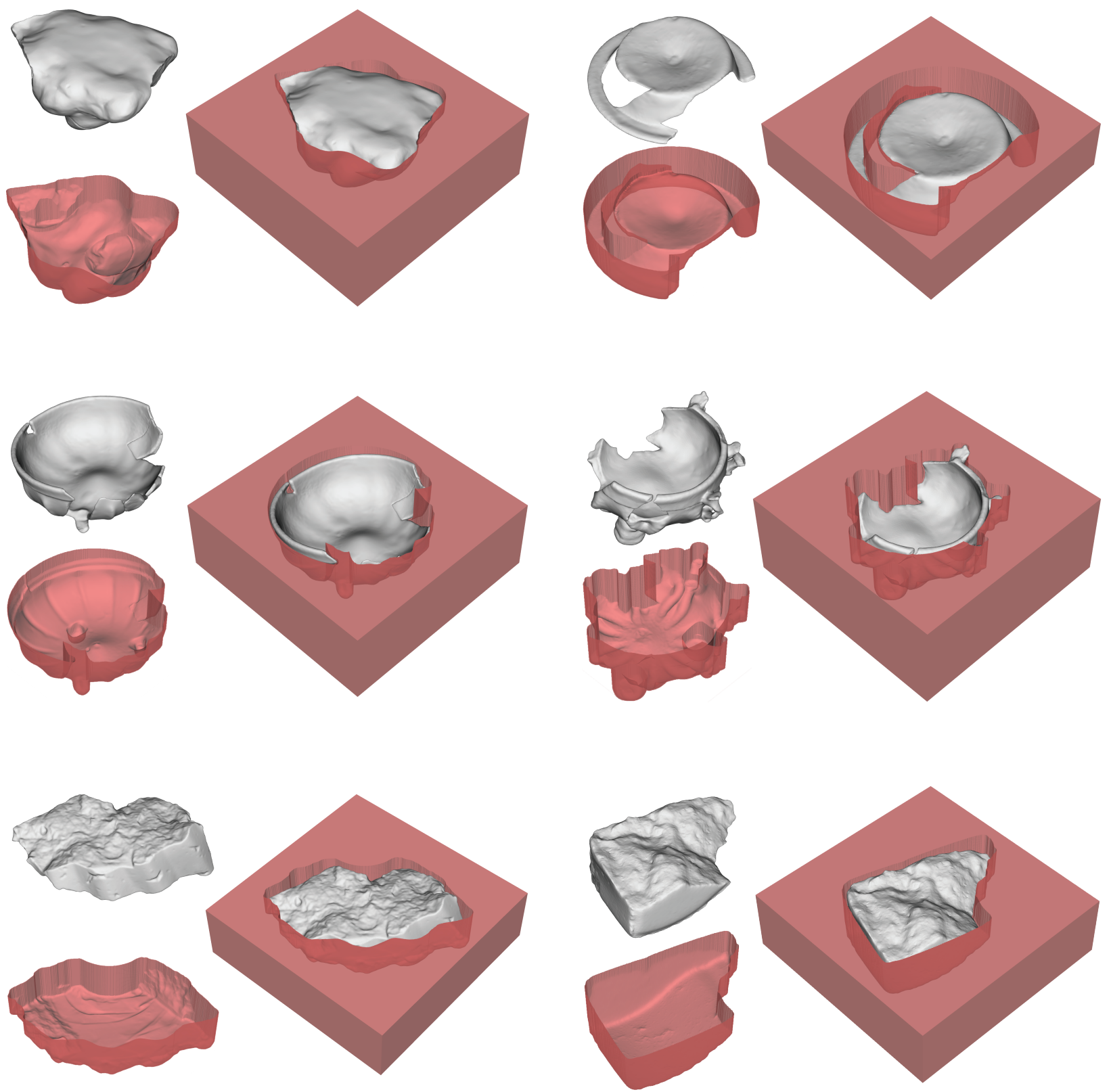

Fig. 6. Achieved result for the automatic pose estimation algorithm. From left to right and top to bottom, artifact names are Venus, Plate, Bowl1, Bowl2, Cake and Brick. For each artifact, upper left image shows its shape, lower left image shows the contact area with the housing and the big right image shows how it fits on the modeled packaging. 3D models of artifacts Venus, Cake and Brick are courtesy by Vienna University of Technology [Vienna University of Technology 2014].

In case a more complex milling strategy is required, imposed by the hardware or by the artifact particularities, the option of exporting a polygonal model of the package is provided, so specialized CAM software can be used. 
Further improvements of the solution should consider different materials for the packaging solution. As different fragile artifacts exist (glass, pottery or even bone tools), different materials for packaging must be used. In this sense, one of the issues to be solved is using appropriate milling cutters or drills according to selected packaging materials. A bad combination of milling tool and CNC parameters (mainly, for high speed CNC processes) could become in bad finishes for the packaging solution.

Moreover, the software application must consider a variety of packaging linings, as packaging for archaeological artifacts usually produce ad-hoc solutions according to the shapes of the objects. This way, packaging solutions must be aligned with different standard possibilities that have to be considered.

\section{ACKNOWLEDGMENT}

This work is supported by the Plan Nacional de I+D+i 2008-2011 from the Ministerio de Economía y Competitividad of Spain, Project ID: HAR2012-38391-C02-01 and HAR2012-38391-C02-02 and the Programa de Ayudas de Investigación y Desarrollo (PAID) of the Universitat Politècnica de València.

Authors would like to thank the Museo de Cerámica de Manises and especially to its director Mrs. Sara Blanes Ibáñez, for providing the artifacts used to illustrate the proposed technique.

\section{REFERENCES}

G. Aboe. 2012. Packaging and storage solutions for archaeological basketry: a selection of practical designs. Journal of the Institute of Conservation 35, 1 (2012), 103-111.

E. Bauer. 1993. Packing museum objects for shipment. Conserve O Gram 17, 2 (July 1993 (1993), 1-4.

S.E. Fulton and S. Rossi-Wilcox. 2008. Harvard's Glass Flowers: a Case Study in Traveling a Fragile Collection. JAIC 47, 1 (January 2008 (2008), 15-26.

K. Gallup and B. Harlow. 2006. Finding solutions to the problems of complex art packing. AIC News 31 (1), $7-8$.

J. Johnson. 1999. NPS Museum Handbook, Part I: Museum Collections. National Park Service Museum Management Program, Washington, DC, Chapter 6: Handling, Packing, and Shipping.

P. J. Marcon. 1991. A Circular Slide Rule for Protective Package Design. Art in Transit: Studies in the Transport of Paintings (1991), 93-106.

P. J Marcon. 2011 (accessed April 13, 2014)b. Six Steps to Safe Shipment. https://www.cci-icc.gc.ca/caringfor-prendresoindes/ articles/sixsteps-sixetapes/index-eng.aspx

P. J Marcon. 2013 (accessed April 13, 2014)a. Agent of Deterioration: Physical Forces. https://www.cci-icc.gc.ca/ caringfor-prendresoindes/articles/sixsteps-sixetapes/index-eng.aspx

P. J. Marcon and T. J. Strang. 1999. PadCAD Cushion design software version 3.0 for windows. In Canadian Conservation Institute. Otawa, Canada.

A. Medeiros-S, K. Rodriguez-Echavarria, M. Griffin, D. Covill, J. Kaminski, and D. B. Arnold. 2012. Parametric 3D-fitted Frames for Packaging Heritage Artefacts.. In VAST, David B. Arnold, Jaime Kaminski, Franco Niccolucci, and Andr Stork (Eds.). Eurographics Association, 105-112. http://dblp.uni-trier.de/db/conf/vast/vast2012.html\#SaRGCKA12

R. Mohamed and A. Mahmoud. 2012. Emphasizing the advantage of 3d printing technology in packaging design development and production in local industries. International Design Journal 1, 1 (2012), 111-119.

C. Murdock and J. S. Johnson. 2001. NPS Museum Handbook, Part I: Museum Collections. National Park Service Museum Management Program, Washington, DC, Chapter Appendix I: Curatorial Care of Archeological Objects.

Massimiliano Pieraccini, Gabriele Guidi, and Carlo Atzeni. 2001. 3D digitizing of cultural heritage. Journal of Cultural Heritage 2, 1 (2001), 63 - 70. http://www.sciencedirect.com/science/article/pii/S1296207401011086

C. Sanchez-Belenguer and E. Vendrell-Vidal. 2012. Archaeological fragment characterization and 3D reconstruction based on projective GPU depth maps. In 18th International Conference on Virtual Systems and Multimedia,VSMM 2012, Milan, Italy, September 2-5, 2012. IEEE, 275-282.

C. Schlichting and Canadian Conservation Institute. 1994. Working with polyethylene foam and fluted plastic sheet. Ottawa : Canadian Conservation Institute (CCI) Technical bulletin no. 14. pp 1-19. Title on added t.p.: Travail de la mousse de polyethylene et des feuilles de plastique cannelees.

Conservation Smithsonian's Digitization Program Office. Smithsonian. 2014 (accessed April 13, 2014). Smithsonian X 3D. http://3d.si.edu

ACM Journal on Computing and Cultural Heritage, Vol. 1, No. 1, Article 1, Publication date: January 1. 
N. Stolow. 1981. Procedures and conservation standards for museum collections in transit and on exhibition / Nathan Stolow. Unesco Paris. 56 pages.

N. Stolow. 1987. Conservation and exhibitions: packing, transport, storage, and environmental consideration. Butterworths London ; Boston. 266 pages.

J. Tetreault and S. Williams. 1993. Guidelines or Selecting Materials for Exhibit, Storage and Transport. In "Conservationally Correct": Realities and Innovations for Exhibitions.

Vienna University of Technology. 2006 (accessed April 20, 2014). 3D Puzzles - Reassembling Fractured Objects by Geometric Matching. http://www.geometrie.tuwien.ac.at/ig/3dpuzzles.html

Received July 2014; revised August 2014; accepted January 2015 